\title{
Low-maintenance energy requirements of obese dogs after weight loss
}

\author{
Alexander J. German ${ }^{1 *}$, Shelley L. Holden ${ }^{1}$, Nicola J. Mather ${ }^{1}$, Penelope J. Morris ${ }^{2}$ and Vincent Biourge ${ }^{3}$ \\ ${ }^{1}$ Department of Obesity and Endocrinology, Institute of Ageing and Chronic Disease, University of Liverpool, Leaburst \\ Campus, Chester High Road, Neston, Wirral CH64 7TE, UK \\ ${ }^{2}$ The Waltham Centre for Pet Nutrition, Waltham-on-the-Wolds, Melton Mowbray, Leicestershire, UK \\ ${ }^{3}$ Royal Canin Research Center, Aimargues, France \\ (Received 20 October 2010 - Revised 23 November 2010 - Accepted 8 December 2010)
}

\section{Abstract}

Weight rebound after successful weight loss is a well-known phenomenon in humans and dogs, possibly due to the fact that energy restriction improves metabolic efficiency, reducing post-weight-loss maintenance energy requirements (MER). The aim of the present study was to estimate post-weight-loss MER in obese pet dogs that had successfully lost weight and did not subsequently rebound. A total of twentyfour obese dogs, successfully completing a weight management programme at the Royal Canin Weight Management Clinic, University of Liverpool (Wirral, UK), were included. In all dogs, a period of $>14 \mathrm{~d}$ of stable weight ( $<1 \%$ change) was identified post-weight loss, when food intake was constant and activity levels were stable (assessed via owners' diary records). Post-weight-loss MER was indirectly estimated by determining dietary energy consumption during this stable weight period. Multivariable linear regression was used to identify factors that were associated with post-weight-loss MER. The mean length of stable weight after weight loss was 54 (SD $34 \cdot 1$ ) d. During this time, MER was 285 (SD 54.8) kJ/kg ${ }^{075}$ per d. The rate of prior weight loss and food intake during the weight-loss phase was positively associated with post-weight-loss MER, while the amount of lean tissue lost was negatively associated with post-weight-loss MER. MER are low after weight loss in obese pet dogs (typically only $10 \%$ more than required during weight-loss MER), which has implications for what should constitute the optimal diet during this period. Preserving lean tissue during weight loss may maximise post-weight-loss MER and help prevent rebound.

Key words: Weight management: Rebound: Canine: Nutrition

Obesity is one of the most common medical disorders in dogs and is linked to both decreased longevity and a variety of associated conditions ${ }^{(1)}$. Management involves reducing energy intake, either using a purpose-formulated weight-loss diet or a drug therapy, and increasing expenditure through increased activity ${ }^{(2,3)}$. However, subsequent weight rebound is common irrespective of the method of weight $\operatorname{loss}^{(3-5)}$. The 'rebound' phenomenon has also been described in human subjects, but its cause is not known. Some researchers $^{(6)}$ have suggested that lower resting metabolic requirements in obese individuals returned to a lean state, and that this predisposes to subsequent weight gain. However, others ${ }^{(7)}$ have suggested that a transient hypothyroid-hypometabolic state develops during energy restriction, which returns to normal during subsequent weight maintenance. The reasons for weight rebound after a weight management regimen in dogs have not been studied in detail, although one experimental study in obese dogs has demonstrated that maintenance energy requirement (MER) decreased significantly as a result of weight loss ${ }^{(4)}$. This finding has recently been confirmed by a study in dogs made experimentally obese by overfeeding, whereby re-induction of obesity occurred more rapidly and with a lower energy intake after a period of weight loss ${ }^{(5)}$. However, equivalent information is lacking in obese pet dogs that have undergone weight management. Furthermore, there has been limited study of the effect on the type of diet fed during weight loss, and, therefore, it is not known whether different dietary strategies may help to prevent rebound. Therefore, the aim of the present study was to estimate MER in dogs post-weight loss and to determine the factors associated with it including animalrelated factors (e.g. sex, age and body composition) and weight-loss factors (e.g. type of diet used, rate of weight loss, percentage weight loss, metabolic energy requirement during weight loss and change in lean mass).

Abbreviations: HPHF, high protein, high fibre; HPMF, high protein, medium fibre; MER, maintenance energy requirement.

*Corresponding author: A. J. German, fax +44 151795 6101, email ajgerman@liv.ac.uk 


\section{Experimental methods}

Dogs

All dogs eligible for inclusion had been referred to the Royal Canin Weight Management Clinic, University of Liverpool, Wirral, UK, for the investigation and management of obesity or obesity-related disorders. Dogs were included in the study if their body weight, food intake and activity levels had remained stable for at least $14 \mathrm{~d}$ during their postweight-loss maintenance phase (described later). The study was performed in adherence to the University of Liverpool Animal Ethics Guidelines and was approved by both the University of Liverpool Research Ethics Committee and the Waltham Ethical Review Committee. The owners of all participating animals gave written informed consent.

\section{Weight-loss regimen}

Full details of the regimen used for weight loss have been described previously ${ }^{(2,8)}$. Briefly, both before and after weight loss, all dogs were confirmed to be systemically well, euthyroid (based upon measurement of free thyroxine by equilibrium dialysis) and had no significant abnormalities on complete blood count, serum biochemical analysis and urinalysis. A tailored weight management protocol was designed for each dog, using either a high-protein, high-fibre (HPHF, Satiety Support; Royal Canin, Aimargues, France) or a highprotein, medium-fibre (HPMF, Obesity Management; Royal Canin) weight-loss $\operatorname{diet}^{(7)}$. Dogs were reassessed regularly throughout the weight-loss programme, and body-weight measurements were taken and changes were made to the dietary plan, if necessary ${ }^{(2,8)}$. Owners weighed all food portions with electronic kitchen scales, and to ensure accuracy of the portion size, a $24 \mathrm{~h}$ ration was first weighed on calibrated electronic scales (Salter, Tonbridge, UK) at the clinic, and then taken home by the client to weigh on their home scales. During the programme, owners maintained a diary where they recorded diet ration fed and activity undertaken. Body composition was determined, both before and after weight loss, by fan-beam dual-energy X-ray absorptiometry (Lunar Prodigy Advance; GE Lunar, Madison, WI, USA) as described previously, allowing the proportion of lean tissue loss to be determined ${ }^{(9)}$.

After reaching their target weight, all dogs continued to attend the clinic for regular weight checks (approximately every 2 weeks) and were switched onto a maintenance regimen, by sequentially increasing food intake in increments of $5-10 \%$ until weight was stable. For the maintenance period, some dogs continued with the diet used during weight loss, while others were switched to commercial maintenance diets, depending upon the preferences of the owner. During the maintenance period, owners continued to maintain their diary covering diet ration fed and activity undertaken.

\section{Determination of a stable maintenance weight period}

To take account of any imprecision from weight measurements, the period of 'stable maintenance weight' was defined as a period, of at least $14 \mathrm{~d}$, starting from the time that they reached their target weight, when there had been $<1 \%$ overall and $<0.2 \% /$ week change in body weight. All weight measurements were made on the same calibrated electronic weigh scales (Soehnle Professional, Murrhardt, Germany).

\section{Determination of post-weight-loss maintenance energy requirement}

Energy intake during the period of stable weight was used to produce an estimate of post-weight-loss MER. The diary records of the owner, for the period in question, were reviewed to verify that daily food intake had remained unchanged, no additional food (i.e. table scraps or treats) had been given and activity levels had been constant (i.e. same daily walk pattern and same daily play activity throughout the period). However, activity levels were not accurately quantified. Daily dietary energy intake was determined from the diary records and the energy content of food, based upon the information provided by the manufacturer. For most diets, the manufacturers had previously measured the metabolisable energy content in animal trials according to the American Association of American Feed Control Officials protocol $^{(10)}$. The only exception was one diet (Chappie; Mars Petcare, Melton Mowbray, UK), where energy content had been calculated using modified Atwater factors ${ }^{(11)}$. Therefore, MER was indirectly estimated by determining dietary energy consumption and is expressed as $\mathrm{kJ} / \mathrm{kg}$ metabolic body weight per $\mathrm{d}\left(\mathrm{kJ} / \mathrm{kg}^{0.75}\right.$ per $\left.\mathrm{d}\right)$.

\section{Data handling and statistical analyses}

All data are expressed as means and standard deviations. To calculate the mean energy intake during weight loss, the sum of the daily energy intakes, while on the diet, was divided by the diet duration. Statistical analyses were performed with a computer software package (Stats Direct version 2.6.2; Stats Direct Limited, Altrincham, UK), with the level of significance set at $P<0.05$ for two-sided analyses. The Shapiro-Wilk test was first used to confirm that all datasets were normally distributed. Paired $t$ tests were used to compare differences in metabolisable energy intake between the start of weight loss, the end of weight loss and the estimated post-weightloss MER. Simple linear regression was used to determine factors associated with differences in post-weight-loss MER. Factors assessed included animal-related factors (e.g. sex, neuter status, age and percentage body fat before weight loss), weight-loss factors (e.g. diet used for weight loss, rate of weight loss, percentage weight loss, metabolic energy intake during weight loss, weight loss duration and change in lean mass) and weight maintenance factors (e.g. duration of the stable weight period). A multivariable linear regression model was constructed, which initially included all variables, but was then refined by backward stepwise elimination of the least significant variable at each round. Variables were retained, in the final model, either if they were significant $(P<0.05)$ or if their removal resulted in a substantial $(\geq 10 \%)$ change to the effect of other variables. 


\section{Results}

\section{Patients and outcome of weight loss}

The age of the twenty-four dogs finally included was 81 (SD 35.6) months; thirteen dogs were male (twelve neutered) and eleven were female (all neutered). The breeds represented included Labrador Retriever ( $n$ 9), mixed breed ( $n$ 3), Cavalier King Charles Spaniel ( $n$ 3), Yorkshire Terrier ( $n$ 3), Cocker Spaniel ( $n$ 2), Border Collie ( $n$ 1), Corgi $(n 1)$, German Shepherd Dog ( $n$ 1) and Golden Retriever ( $n$ 1). During weight loss, fourteen dogs were fed the HPHF diet, and the remaining ten dogs were fed the HPMF diet. The duration of the weight-loss period was 222 (SD 129.8) d, and dogs lost 23.6 (SD 8.52 ) \% of starting body weight over this time (mean rate of loss 0.9 (SD 0.32 ) \% of starting body weight/ week). Metabolisable energy intake at the start of weight loss (i.e. the initial allocation used for weight loss) was 268 (sD $35 \cdot 2$ ) $\mathrm{kJ} / \mathrm{kg}^{0.75}$ per $\mathrm{d}$, metabolisable energy intake at the end of weight loss was 251 (SD 34.8) kJ/ $\mathrm{kg}^{0.75}$ per $\mathrm{d}$ and

드 mean metabolisable intake over the whole of the weightloss period was 255 (SD $32 \cdot 2$ ) $\mathrm{kJ} / \mathrm{kg}^{0.75}$ per d. Metabolisable energy intake at the end of weight loss was significantly lower than at the start $(P=0.007)$. As determined by dualenergy X-ray absorptiometry, lean tissue mass comprised 12 (sD $16 \cdot 5) \%$ of the weight lost.

\section{Estimated post-weight-loss maintenance energy requirement}

The duration of the stable weight period was 54 (SD 34.1) d and mean maintenance weight was $24 \cdot 2$ (SD 12.60) kg. During this period, overall weight change was -0.2 (SD $0.32) \%$, while weight change per week was 0.02 (SD $0.053) \%$. For the maintenance period, twelve and seven dogs continued with the HPHF and HPMF diets, respectively. The remaining dogs were fed standard dry maintenance diets (one each of the following: Chappie, Mars Petcare; Neutered and stone diet, Royal Canin; Neutered large adult diet, Royal Canin; Medium mature diet, Royal Canin; Labrador diet, Royal Canin). Estimated post-weight-loss MER was 285 (SD $54.8) \mathrm{kJ} / \mathrm{kg}^{0.75}$ per $\mathrm{d}$, which was significantly higher than metabolisable energy intake at the end of the weight-loss

Table 1. Associations between post-weight-loss metabolic energy requirement and weight loss variables using multivariable linear regression

\begin{tabular}{llll}
\hline Multivariable regression & $R$ & $R^{2}$ & \multicolumn{1}{c}{$P$} \\
\hline Final model & 0.74 & 0.55 & 0.003 \\
Diet used during weight loss ${ }^{*}$ & 0.37 & 0.14 & $0.098 \dagger$ \\
Rate of weight loss & 0.57 & 0.32 & 0.008 \\
Mean ME intake during weight loss & 0.56 & 0.31 & 0.008 \\
Proportion of lean tissue loss & -0.40 & 0.16 & $0.068+$ \\
\hline ME, metabolisable energy. & & & \\
* Based upon a dummy variable where dogs fed the high-protein, medium-fibre diet \\
were assigned a value of 0 and dogs fed the high-protein, high-fibre diet were \\
assigned a value of 1. \\
t Retained in the final model as their removal resulted in a substantial $(\geq 10 \%)$ \\
change to the effect of other variables.
\end{tabular}

period $(P<0 \cdot 001)$ but not significantly different from intake at the start of weight loss $(P=0 \cdot 118)$.

\section{Factors associated with estimated post-weight-loss maintenance energy requirement}

On multivariable analysis, post-weight-loss MER was positively correlated with both the rate of weight loss and metabolisable energy intake during weight loss (both increased as post-weight-loss MER increased; Table 1). Furthermore, diet type was positively correlated (MER was higher when the HPHF diet was used for weight loss; $P=0.098$ ) and the proportion of weight lost as lean tissue was negatively correlated with MER during the period of stable weight (post-weight-loss MER was higher when lean tissue loss was lower; Table 1).

\section{Discussion}

In the present study, we have estimated the post-weight-loss MER of obese pet dogs after completing a weight-loss programme and attaining ideal body condition. Most notably, mean post-weight-loss MER was low in this group, approximately $10 \%$ above the mean metabolisable energy intake during the weight-loss period, and this may explain the propensity for rebound in dogs after weight loss ${ }^{(3-5)}$. Given that none of the owners had kept accurate records of food intake before weight loss, it was not possible to determine whether these requirements were lower as a result of the weight loss. Therefore, it is unclear as to whether the findings represent an inherently low MER in this population, which might have predisposed them to obesity. However, the fact that post-weight-loss MER was not significantly different from the level of metabolisable energy intake that initially induced weight loss would suggest that MER had decreased as a result of the dietary energy restriction. This finding is consistent with previous experimental studies in dogs, where post-weight-loss MER were significantly lower than MER before weight loss ${ }^{(4,5)}$.

The main practical concern with such a low post-weightloss MER would be that the daily meal allocation would invariably be modest, when fed a standard canine maintenance diet, and this may not produce adequate satiety predisposing to increased food-seeking behaviour. Furthermore, standard maintenance diets are designed to be complete and balanced when fed at typical maintenance energy levels for adult dogs. However, the average daily metabolisable energy intake after weight loss, in dogs of the present study, was $285 \mathrm{~kJ} / \mathrm{kg}^{0.75}$ per $\mathrm{d}$, and some dogs needed as little as $218 \mathrm{~kJ} / \mathrm{kg}^{0.75}$ per $\mathrm{d}$, which is considerably lower than the current MER recommendation for inactive adult pet dogs $\left(400-440 \mathrm{~kJ} / \mathrm{kg}^{0.75} \text { per } \mathrm{d}\right)^{(11)}$. When fed long-term, this could lead to the development of nutrient deficiencies, and, for this reason, it may be preferable to feed a diet during the maintenance period which contains increased levels of protein, vitamins and minerals relative to energy content. Weight-loss diets are formulated in such a way, and usually also have an altered macronutrient content profile (i.e. high 
protein and high fibre), which improves satiety in dogs ${ }^{(12)}$. However, the additional cost and inconvenience (in that such diets must usually be purchased from a veterinary surgeon) may mean that owners are reluctant to continue to feed a purpose-formulated weight-loss diet lifelong.

Post-weight-loss MER varied greatly among dogs. This partly reflects the inherent variation in an outbred population but is also influenced by various characteristics of the weightloss regimen including the amount of lean tissue lost. This finding is logical, given that lean tissue is more metabolically active than adipose tissue, but suggests that post-weight-loss MER could be maximised if lean tissue loss can be prevented. For instance, incorporating exercise in weight-loss programmes is known to preserve lean tissue mass during weight loss in human subjects ${ }^{(13)}$.

The present study has a number of important limitations. First, given the fact that the study involved pet dogs, there were ethical limitations on the procedures that could be used meaning that more invasive techniques, such as the use of indirect calorimetry and isotopic methods ${ }^{(14)}$, could not be used. Such techniques are undoubtedly more accurate and would, perhaps, have improved accuracy of the results. Instead, we estimated energy requirement indirectly, using diary records completed by the owner, and such methods are prone to under-reporting ${ }^{(15)}$. Other inaccuracies would have arisen from variability in activity levels and inaccuracy in portion allocation, neither of which can be guaranteed when relying on diary records. Therefore, the estimates of energy requirement must be taken with caution and, ideally, need verification with further studies. The main advantage of adopting our approach was the fact that a population of pet dogs could be studied, rather than a colony of research dogs.

In summary, the present study showed that the post-weightloss MER of obese dogs after a weight-loss regimen is low, and this has implications on maintaining a dog at its optimum condition after weight loss. Further work is required to determine what implications this may have in terms of development of nutrient deficiencies when dogs with lower than average energy requirements are fed diets designed for maintenance of dogs with normal energy requirements.

\section{Acknowledgements}

The following conflicts of interest apply: Royal Canin manufactured the diets used in the present study; V. B. is an employee of Royal Canin; P. J. M. is an employee of Mars Petcare; Royal Canin financially supports the posts of A. J. G. and S. L. H., at the University of Liverpool. The present study was funded by grants from Royal Canin and Mars Petcare. The contributions of the authors were as follows:
A. J. G. participated in the study design, data collection, analysis and interpretation of the data and writing of the manuscript; S. L. H. and N. J. M. were involved in the data collection, analysis and interpretation and reviewing the manuscript; P. J. M. and V. B. conducted the data analysis and interpretation, and helped in reviewing the manuscript.

\section{References}

1. German AJ (2006) The growing problem of obesity in dogs and cats. J Nutr 136, 1940S-1946S.

2. German AJ, Holden SL, Bissot T, et al. (2007) Dietary energy restriction and successful weight loss in obese client-owned dogs. J Vet Intern Med 21, 1174-1180.

3. Gossellin J, Peachey S, Sherington J, et al. (2007) Evaluation of dirlotapide for sustained weight loss in overweight Labrador retrievers. J Vet Pharmacol Ther 30, 55-65.

4. Laflamme DP \& Kuhlman G (1995) The effect of weight loss regimen on subsequent weight maintenance in dogs. Nutr Res 15, 1019-1028.

5. Nagaoka D, Mitsuhashi Y, Angell R, et al. (2010) Re-induction of obese body weight occurs more rapidly and at lower caloric intake in beagles. I Anim Physiol Anim Nutr 94, 287-292.

6. Astrup A, Gotzsche PC, van de Werken K, et al. (1999) Metaanalysis of resting metabolic rate in formerly obese subjects. Am J Clin Nutr 69, 1117-1122.

7. Weinsier RL, Nagy TR, Hunter GR, et al. (2000) Do adaptive changes in metabolic rate favor weight regain in weightreduced individuals? An examination of the set-point theory. Am J Clin Nutr 72, 1088-1094.

8. German AJ, Holden SL, Bissot T, et al. (2010) A high protein high fibre diet improves weight loss in obese dogs. Vet J 183, 294-297.

9. Raffan E, Holden SL, Cullingham F, et al. (2006) Standardized positioning is essential for precise determination of body composition using dual-energy X-ray absorptiometry in dogs. J Nutr 136, 1976S-1978S.

10. Association of American Feed Control Officials (2010) Dogs and cats nutrient profiles. In AAFCO Official Publication, pp. 169-183. Washington, DC: AAFCO.

11. National Research Council (NRC) (2006) Nutrient Requirements of Dogs and Cats. Washington, DC: National Academy Press.

12. Weber M, Bissot T, Servet E, et al. (2007) A high protein, high fiber diet designed for weight loss improves satiety in dogs. $J$ Vet Intern Med 21, 1203-1208.

13. Ross R, Pedwell $\mathrm{H}$ \& Rissanen J (1995) Effects of energy restriction and exercise on skeletal-muscle and adipose-tissue in women as measured by magnetic-resonance-imaging. Am J Clin Nutr 61, 1179-1185.

14. Pouteau EB, Mariot SM, Martin LJ, et al. (2002) Rate of carbon dioxide production and energy expenditure in fed and food-deprived adult dogs as determined by indirect calorimetry and isotopic methods. Am J Vet Res 63, 111-118.

15. Heitmann BL, Lissner L \& Osler M (2000) Do we eat less fat, or just report so? Int J Obes 24, 435-442. 